Concentrations of iron in the joint tissues as high as occur in haemochromatosis are also found in rheumatoid arthritis, though it is believed here that the deposits arise from the continual oozing of blood from vascular granulation tissue. ${ }^{8}$ The iron lies deep in the synovium, which shows proliferation and infiltration by inflammatory cells, whereas in haemochromatosis the deposits of iron are found in the cells of the synovial lining and the underlying stroma appears undamaged.

Osteoporosis may also occur. Schumacher ${ }^{1}$ found it in the spine in 6 out of 23 patients and F. Delbarre ${ }^{9}$ described some severe cases. However, Wardle and Patton ${ }^{2}$ noted osteoporosis in only 2 of 13 spines examined, though localized osteoporosis of the hands was evident in 7 . Two of their patients had hypercalciuria, but the exact cause of the osteoporosis is uncertain. H. C. Seftel and colleagues ${ }^{10}$ from South Africa have described spinal osteoporosis in the Bantu in association with severe siderosis. The siderosis and osteoporosis were also closely associated with deficiency of ascorbic acid, and they postulated that it was the latter which led to the diminished formation of bone. The large storage deposits of iron, which are in the ferric form, represent a massive oxidation potential, and part of the available ascorbic acid may be irreversibly oxidized by these deposits. A contributing factor in the Bantu is deficient dietary intake, and similar though less severe changes have been noted in idiopathic haemochromatosis and transfusional siderosis. ${ }^{11}$ Another important factor in the osteoporosis may be deficiency of androgen. Hypogonadism is a well-known feature of haemochromatosis, and Wardle and Patton found low levels of urinary 17oxosteroid and 17-oxogenic steroids in their patients.

Since venesection will not improve the arthropathy, and since prevention appears unlikely, what can be done to help these patients? In those with relatively minor symptoms aspirin or one of the anti-inflammatory agents such as phenylbutazone or indomethacin can be of considerable help. Acute attacks of arthritis due to synovitis require immobilization of the joint with aspiration of the joint fluid. This often gives relief as well as enabling confirmation of the diagnosis by examination of the joint fluid by polarized-light microscopy. Severe painful arthritis of the hip can be helped by an osteotomy and other surgical procedures.

\section{Motor Neurone Disease and Neuronal Metabolism}

The aetiology of motor neurone disease is unknown. Whether the disease presents clinically with progressive wasting of voluntary muscles in the limbs (progressive muscular atrophy), with less evident muscular atrophy and fasciculation but with predominant signs of degeneration in the corticospinal tracts (amyotrophic lateral sclerosis), or whether the motor nuclei of the cranial nerves and the corticospinal tracts in the brain stem are predominantly involved (progressive bulbar palsy), there is little or no evidence to indicate the nature of the disease process in the upper or lower motor neurones which accounts for or accompanies their degeneration.

One obstacle to studies of neuronal metabolism in this disease is that, whereas in primary muscle disease the metabolism of the muscle fibre can be studied by biopsy, this cannot be used for studying changes in the anterior horn cells of the spinal cord. The recent description of a genetically determined form of lower motor neurone degeneration in the mouse ${ }^{1}$ may mean that an animal has been found on which biochemical and electrophysiological studies may be possible in the future. But it is uncertain whether this mouse disease is strictly comparable with the human disorder.

In 1957 E. W. Poole ${ }^{2}$ reported that ischaemic and postischaemic paraesthesiae were absent in the limbs of patients with motor neurone disease even after periods of arterial occlusion which constantly produced such phenomena in normal persons. It is a well-known clinical finding that patients with motor neurone disease show no subjective or objective sensory abnormality, so that Poole's ${ }^{2}$ findings were difficult to explain. B. Shahani and W. Ritchie Russell ${ }^{3}$ have now reinvestigated this phenomenon in three patients suffering from motor neurone disease and in three control persons. They were stimulated to do so by the findings of G. Gregerson $^{4}$ and K. N. Seneviratne and O. Peiris, ${ }^{5}$ who showed that peripheral nerves in cases of diabetes may be unusually resistant to ischaemia. They have studied compound motor action potentials ( $M$-waves) recorded from the abductor pollicis brevis muscle during supramaximal stimulation of the median nerve and $\mathrm{H}$-waves (reflex responses obtained during submaximal stimulation), as well as $M$-waves recorded from one soleus during stimulation of the medial popliteal nerve. These observations were carried out in their cases and in controls before and after arterial occlusion produced by the application of a sphygmomanometer cuff to the upper arm or thigh at a pressure greater than the patient's systolic arterial blood pressure for periods of 15-20 minutes. The authors have confirmed that ischaemic and postischaemic paraesthesiae are either absent or mild in patients with motor neurone disease, and from the results of their observations they have concluded that the absence of such paraesthesiae in this condition can be related to a relatively high resistance of large sensory nerve fibres to ischaemia. They conclude that this abnormality of peripheral nerves may indicate that in motor neurone disease neuronal metabolism is altered.

These preliminary results need to be confirmed in studies of larger groups of patients. Moreover, it should be possible to show that sensory fibres are abnormally resistant to ischaemia by methods utilizing direct stimulation and recording from them. It is important too that the sensitivity of the motor nerve fibres to ischaemia should be studied in patients with motor neurone disease; inspection of the $M$-waves illustrated by Shahani and Ritchie Russell ${ }^{3}$ suggests that the sensitivity of these fibres in patients was probably similar to that in control subjects. Hence, though these observations are of interest, and though studies of the behaviour of peripheral nerve fibres in patients with motor neurone disease may perhaps allow us to draw valid conclusions about the behaviour of the whole neurone, the finding that sensory nerve fibres in this condition are abnormally resistant to ischaemia can be regarded as no more than a first step along a road beset with many difficulties.

1 Duchen, L. W., and Strich, S. J., Fournal of Neurology, Neurosurgery and Psychiatry, 1968, 31, 535.

2 Poole, E. W., Fournal of Neurology, Neurosurgery and Psychiatry, 1957, 20, 225.

- Shahani, B., and Russell, W. R., Fournal of Neurology, Neurosurgery and Psychiatry, 1969, 32, 1.

- Gregerson, G., fournal of Neurology, Neurosurgery and Psychiatry, 1968, 31, 175 .

- Seneviratne, K. N., and Peiris, O. A., Fournal of Neurology, Neurosurgery and Psychiatry, 1968, 31, 338. 